PROCEEDINGS OF THE

AMERICAN MATHEMATICAL SOCIETY

Volume 126, Number 12, December 1998, Pages 3521-3526

S 0002-9939(98)04952-1

\title{
SEMIGROUP PROPERTIES OF FACTORS IN THE POLAR DECOMPOSITION OR THE OPERATOR DE-MOIVRE FORMULA
}

\author{
XIMENA CATEPILLÁN AND WACŁAW SZYMAŃSKI \\ (Communicated by Palle E. T. Jorgensen)
}

\begin{abstract}
We give necessary and sufficient conditions under which the factors in the polar decomposition of a semigroup homomorphism are themselves semigroup homomorphisms.
\end{abstract}

\section{INTRODUCTION}

For a complex number $z$ its polar form is $z=|z| e^{i \theta}$ with a real number $\theta$. The polar decomposition of a bounded Hilbert space operator $T=V|T|$, where $|T|=\left(T^{*} T\right)^{1 / 2}$ and $V$ is an appropriate partial isometry, is the non-commutative generalization of the polar form of a complex number. $\mathbb{N}$ is the additive semigroup of natural numbers.

De-Moivre's formula says that $z^{n}=|z|^{n} e^{i n \theta}, n \in \mathbb{N}$.

It is therefore a natural question to ask:

When does $T^{n}=V^{n}|T|^{n}, n \in \mathbb{N}$, for an operator $T$ with the polar decomposition $T=|T| V ?$

A sufficient condition is, clearly, that $T$ is quasinormal (see definition below). From general results of this paper it will follow that quasinormality is also necessary.

The above question is equivalent to:

When does $\left|T^{n}\right|=|T|^{n}$ and the partial isometric factor of $T^{n}$ equal $V^{n}$ ?

Upon a closer examination, this question can be formulated using the semigroup language as follows: The mapping $n \rightarrow T^{n}, n \in \mathbb{N}$, is, obviously, a semigroup homomorphism. Hence our question is equivalent to the question: When are the mappings $n \rightarrow\left|T^{n}\right|$ and $n \rightarrow$ (the partial isometric factor of $T^{n}$ ) semigroup homomorphisms?

The only answer in this context has been given by B. Morrel and P. Muhly in [4], Theorem I, who proved that if the operator $T$ is centered (see definition below), then the partial isometric factor of $T^{n}$ is $V^{n}$. Their proof uses the principle of mathematical induction and thus cannot be generalized to any other semigroup.

Before we state the general problem let us introduce some terminology.

$B(H)$ is the algebra of all linear, bounded operators in a Hilbert space $H . I$ denotes the identity operator on $H$. An operator $V \in B(H)$ is called a partial isometry if $V^{*} V$ is an orthogonal projection, or, equivalently, if $V V^{*} V=V$. By

Received by the editors January 21, 1997.

1991 Mathematics Subject Classification. Primary 47B20, 47D03.

(C) 1998 American Mathematical Society 
the polar decomposition of $T \in B(H)$ we understand the decomposition $T=V|T|$ with $|T|=\left(T^{*} T\right)^{1 / 2}$ and $V: \overline{R(|T|)} \rightarrow \overline{R(T)}, V|T| x=T x, x \in H$, $\operatorname{ker} T=\operatorname{ker} V$. $T \in B(H)$ is quasinormal if $T^{*} T$ commutes with $T$ or, equivalently, if the factors in the polar decomposition of $T$ commute. $T \in B(H)$ is called centered if the set $\left\{T^{n}\left(T^{*}\right)^{n},\left(T^{*}\right)^{m} T^{m}, n, m \in \mathbb{N}, n, m \neq 0\right\}$ is commutative. $T \in B(H)$ is hyponormal if $T^{*} T-T T^{*} \geq 0$.

Let $S$ be a commutative semigroup with 1 .

A mapping $\pi: S \rightarrow B(H)$ is called a semigroup homomorphism if $\pi(s+t)=$ $\pi(s) \pi(t), s, t \in S$, and $\pi(1)=I$, a normal homomorphism if the set $\left\{\pi(s), \pi(t)^{*}\right.$, $s, t \in S\}$ is commutative, a quasinormal homomorphism if the set $\left\{\pi(s)^{*} \pi(s), \pi(t)\right.$, $s, t \in S\}$ is commutative, a subnormal homomorphism if there is a Hilbert space $K$ containing $H$ and a normal homomorphism $\tau: S \rightarrow B(H)$ such that $H$ is invariant for each $\tau(s)$ and $\tau(s)_{\mid H}=\pi(s), s \in S$, and a centered homomorphism if the set $\left\{\pi(s)^{*} \pi(s), \pi(t) \pi(t)^{*}, s, t \in S\right\}$ is commutative. All these special kinds of homomorphisms are, clearly, assumed to be semigroup homomorphisms. Clearly, each quasinormal homomorphism is centered.

Let $\pi: S \rightarrow B(H)$ be a semigroup homomorphism. Let $\pi(s)=\mu(s) \theta(s)$ be the polar decomposition of the operator $\pi(s), s \in S$.

In this paper we will answer the question under which conditions $\mu$ and $\theta$ are semigroup homomorphisms. This result applied to $S=\mathbb{N}$ will answer our first question, and Theorem 1 applied to the semigroup $[0, \infty)$ will generalize a result of M. Embry in [2], Theorem 6, which she proved under the additional assumption of strong continuity.

\section{The RESUlts}

Let $S$ be a commutative semigroup with 1 .

Let $\pi: S \rightarrow B(H)$ be a semigroup homomorphism, and let $\pi(s)=\theta(s) \mu(s)$ be the polar decomposition of $\pi(s), s \in S$. $E(t)=\theta(t)^{*} \theta(t)$ is the initial projection for $\theta(t)$ and $F(t)=\theta(t) \theta(t)^{*}$ is the final projection for $\theta(t)$.

It is known in general that

$$
\theta(t) \theta(t)^{*} \theta(t)=\theta(t),
$$

$\theta(t)^{*} \theta(t)$ is the projection onto $(\operatorname{ker} \pi(t))^{\perp}=(\operatorname{ker} \mu(t))^{\perp}=\overline{\operatorname{ran} \mu(t)}$, and

$$
\theta(t)^{*} \theta(t) \mu(t)=\mu(t) .
$$

Theorem 1. (a) If $\pi$ is centered, then $\theta$ is a semigroup homomorphism.

(b) Assume additionally that for each $s, t \in S$ there exists $r \in S$ such that $s=t+r$ or $t=s+r$. If $\theta$ is a semigroup homomorphism, then $\pi$ is centered.

Proof. (a) As in the case of one operator, $\mu(s), E(t), F(r)$ commute for all $s, t, r \in S$ (cf. [4]).

$$
\mu(t) \text { and } \theta(r) \mu(r) \theta(r)^{*} \text { commute, }
$$

because $\mu(t)^{2}=\pi(t)^{*} \pi(t)$ and $\theta(r) \mu(r)^{2} \theta(r)^{*}=\pi(r) \pi(r)^{*}$ commute.

Notice that

$$
\theta(r) \mu(r) \theta(r)^{*}=\left(\theta(r) \mu(r)^{2} \theta(r)^{*}\right)^{1 / 2} .
$$

Moreover,

$$
\theta(t+r) \mu(t+r)=\pi(t+r)=\pi(t) \pi(r)=\theta(t) \mu(t) \theta(r) \mu(r) .
$$


Since $E(t+r)$ commutes with $\mu(t+r)$ and $E(t+r) \leq E(r)$ because $\operatorname{ran} \mu(t+r) \subset$ $\operatorname{ran} \mu(r)$, it follows that

$$
\mu(t+r)=\mu(t+r) E(t+r)=\mu(t+r) E(t+r) E(r)=\mu(t+r) E(r),
$$

and by using (3) and (2) we get that

$$
\begin{aligned}
\theta(t+r) \mu(t+r) & =\theta(t+r) \mu(t+r) E(r)=\theta(t+r) \mu(t+r) \theta(r)^{*} \theta(r) \\
& =\theta(t) \mu(t) \theta(r) \mu(r) \theta(r)^{*} \theta(r)=\theta(t) \theta(r) \mu(r) \theta(r)^{*} \mu(t) \theta(r) .
\end{aligned}
$$

Thus,

$$
\theta(t+r) \mu(t+r)=\theta(t) \theta(r)\left[\mu(r) \theta(r)^{*} \mu(t) \theta(r)\right] .
$$

We claim that the right hand side in this equality is the polar decomposition of $\pi(t+r)$.

First let us prove that $\mu(r)$ and $\theta(r)^{*} \mu(t) \theta(r)$ commute. Using (1) and (2) we get

$$
\begin{aligned}
\mu(r) \theta(r)^{*} \mu(t) \theta(r) & =\theta(r)^{*} \theta(r) \mu(r) \theta(r)^{*} \mu(t) \theta(r) \\
& =\theta(r)^{*} \mu(t) \theta(r) \mu(r) \theta(r)^{*} \theta(r)=\theta(r)^{*} \mu(t) \theta(r) \mu(r) .
\end{aligned}
$$

Consequently,

$$
\mu(r) \theta(r)^{*} \mu(t) \theta(r) \geq 0
$$

Moreover,

$$
\left(\mu(r) \theta(r)^{*} \mu(t) \theta(r)\right)^{2}=\pi(t+r)^{*} \pi(t+r),
$$

because

$$
\begin{aligned}
\mu(r) & \theta(r)^{*} \mu(t) \theta(r) \mu(r) \theta(r)^{*} \mu(t) \theta(r) \\
& =\mu(r) \theta(r)^{*} \mu(t) \theta(r) \theta(r)^{*} \mu(t) \theta(r) \mu(r)=\pi(r)^{*} \mu(t)^{2} \theta(r) \theta(r)^{*} \theta(r) \mu(r) \\
& =\pi(r)^{*} \mu(t)^{2} \theta(r) \mu(r)=\pi(r)^{*} \pi(t)^{*} \pi(t) \pi(r)=\pi(t+r)^{*} \pi(t+r) .
\end{aligned}
$$

Hence,

$$
\mu(r) \theta(r)^{*} \mu(t) \theta(r)=|\pi(t+r)|=\mu(t+r) .
$$

It remains to be proved that $\theta(t) \theta(r)=\theta(t+r)$. Since $E(t), F(r)$ commute, $\theta(t) \theta(r)$ is a partial isometry. Once it is proved that $\operatorname{ker} \pi(t+r)=\operatorname{ker} \theta(t) \theta(r)$, the uniqueness of the polar decomposition will finish the proof. Since ker $\pi(t+r)=$ ker $\mu(t+r)$ and $\mu(t+r)=\mu(r) \theta(r)^{*} \mu(t) \theta(r)$ as shown above, taking into account that $\operatorname{ker} \mu(s)=\operatorname{ker} \theta(s), s \in S$, we get $(x \in H)$

$$
\begin{aligned}
& \mu(r) \theta(r)^{*} \mu(t) \theta(r) x=0 \Leftrightarrow \theta(r) \theta(r)^{*} \mu(t) \theta(r) x=0 \\
& \quad \Leftrightarrow \mu(t) \theta(r) \theta(r)^{*} \theta(r) x=0 \Leftrightarrow \mu(t) \theta(r) x=0 \Leftrightarrow \theta(t) \theta(r) x=0 .
\end{aligned}
$$

(b) Let $r, t \in S$. Then

$$
\theta(t+r) \mu(t+r)=\pi(t+r)=\pi(t) \pi(r)=\theta(t) \mu(t) \theta(r) \mu(r) .
$$

Multiplying on the left by $\theta(t)^{*}$ and using (1), we get

$$
\theta(t)^{*} \theta(t+r) \mu(t+r)=\mu(t) \theta(r) \mu(r) .
$$

On the other hand, $\theta$ is assumed to be a homomorphism. Therefore,

$$
\begin{aligned}
\theta(t)^{*} \theta(t+r) & =\theta(t)^{*} \theta(t) \theta(r)=E(t) \theta(r) \theta(r)^{*} \theta(r)=E(t) F(r) \theta(r)=F(r) E(t) \theta(r) \\
& =\theta(r) \theta(r)^{*} \theta(t)^{*} \theta(t) \theta(r)=\theta(r) \theta(t+r)^{*} \theta(t+r) .
\end{aligned}
$$


If we substitute this into (4) and use (1) for $t+r$, we get

$$
\theta(r) \mu(t+r)=\mu(t) \theta(r) \mu(r) .
$$

Now, multiplying on the right by $\theta(r)^{*}$ we obtain

$$
\theta(r) \mu(t+r) \theta(r)^{*}=\mu(t)\left[\theta(r) \mu(r) \theta(r)^{*}\right] .
$$

Since the operator on the left hand side, $\mu(t)$, and $\theta(r) \mu(r) \theta(r)^{*}$ are all selfadjoint, we conclude that $\mu(t)$ commutes with $\theta(r) \mu(r) \theta(r)^{*}$.

Therefore if we multiply the equality (5) on the left by $\theta(r)^{*}$ and use the inclusion $\operatorname{ran} \mu(t+r) \subset \operatorname{ran} \mu(r)$, we get

$$
\mu(t+r)=\theta(r)^{*} \theta(r) \mu(t+r)=\theta(r)^{*} \mu(t) \theta(r) \mu(r) .
$$

Therefore, since $\mu(t+r)$ is self-adjoint, $\mu(r)$ commutes with $\theta(r)^{*} \mu(t) \theta(r)$. Finally,

$$
\mu(t+r) \mu(r)=\theta(r)^{*} \mu(t) \theta(r) \mu(r)^{2}=\mu(r) \theta(r)^{*} \mu(t) \theta(r) \mu(r)=\mu(r) \mu(t+r) .
$$

Hence, by our additional assumption in (b),

$$
\mu(t) \mu(s)=\mu(s) \mu(t), \quad s, t \in S .
$$

Now we are ready to prove the claim, i.e., three commutativity relations. The proofs follow.

First:

$$
\pi(s)^{*} \pi(s) \pi(t)^{*} \pi(t)=\mu(s)^{2} \mu(t)^{2}=\mu(t)^{2} \mu(s)^{2}=\pi(t)^{*} \pi(t) \pi(s)^{*} \pi(s) .
$$

Second:

$$
\begin{aligned}
& \pi(t)^{*} \pi(t) \pi(r) \pi(r)^{*}=\mu(t)^{2} \theta(r) \mu(r)^{2} \theta(r)^{*}=\mu(t)[\mu(t) \theta(r) \mu(r)] \mu(r) \theta(r)^{*} \\
& \quad=\mu(t) \theta(r) \mu(t+r) \mu(r) \theta(r)^{*}=[\mu(t) \theta(r) \mu(r)] \mu(t+r) \theta(r)^{*}=\theta(r) \mu(t+r)^{2} \theta(r)^{*} .
\end{aligned}
$$

Since the last operator is self-adjoint, $\pi(t)^{*} \pi(t)$ commutes with $\pi(r) \pi(r)^{*}$.

Third:

$$
\begin{aligned}
\pi(t+ & r) \pi(t+r)^{*} \pi(t) \pi(t)^{*} \\
& =\theta(t+r) \mu(t+r)^{2} \theta(t+r)^{*} \theta(t) \mu(t)^{2} \theta(t) \\
& =\theta(t+r) \mu(t+r)^{2} \theta(r)^{*} \theta(t)^{*} \theta(t) \mu(t)^{2} \theta(t) \\
& =\theta(t+r) \mu(t+r)^{2} \theta(r)^{*} \mu(t)^{2} \theta(t)^{*} \\
& =\theta(t) \theta(r) \mu(t+r) \mu(t+r) \theta(r)^{*} \mu(t)^{2} \theta(t)^{*} \\
& =\theta(t) \mu(t) \theta(r) \mu(r) \mu(t+r) \theta(r)^{*} \mu(t)^{2} \theta(t)^{*} \\
& =\theta(t) \mu(t) \theta(r) \mu(t+r) \mu(r) \theta(r)^{*} \mu(t)^{2} \theta(t)^{*} \\
& =\theta(t) \mu(t) \mu(t) \theta(r) \mu(r) \mu(r) \theta(r)^{*} \mu(t)^{2} \theta(t)^{*} \\
& =\theta(t) \mu(t)^{2} \theta(r) \mu(r)^{2} \theta(r)^{*} \mu(t)^{2} \theta(t)^{*} .
\end{aligned}
$$

Since the last operator is self-adjoint, $\pi(t+r) \pi(t+r)^{*}$ commutes with $\pi(t) \pi(t)^{*}$. Using the additional assumption in (b) we get that $\pi(s) \pi(s)^{*}$ commutes with $\pi(t) \pi(t)^{*}$. 

$[2]$.

Our proof of part (b) follows the idea of the proof of Theorem 6 of M. Embry in

In part (b) we made an additional assumption about the semigroup $S$. Unfortunately, we were not able to prove part (b) without that assumption, which not all semigroups satisfy, e.g., $\mathbb{N} \times \mathbb{N}$ does not. This assumption has a certain order-type flavor, which is, perhaps, essential.

Theorem 2. Let $S$ be a commutative semigroup with unit and let $\pi: S \rightarrow B(H)$ be a semigroup homomorphism. $\pi$ is a quasinormal homomorphism if and only if $\mu$ is a semigroup homomorphism.

Proof. Suppose $\pi$ is a quasinormal homomorphism. Notice that $\theta(t), \mu(t), \theta(s)$, and $\mu(s)$ commute, $s, t \in S$.

Thus $\pi(s+t)=\pi(s) \pi(t)=\theta(s) \mu(s) \theta(t) \mu(t)=\theta(s) \theta(t) \mu(s) \mu(t)$. Now, since $\pi$ is quasinormal, it is centered and therefore $\theta$ is also a homomorphism by Theorem 1 . Hence, $\pi(t+s)=\theta(s+t) \mu(s) \mu(t)$ and by the uniqueness of the polar decomposition we get that $\mu(s+t)=\mu(s) \mu(t)$. This part of the proof can essentially be found in A. Lubin [3], proof of Theorem 4.5.

Suppose now that $\mu$ is a semigroup homomorphism. To prove that $\pi$ is quasinormal we use one of the equivalent conditions for quasinormality from Theorem 3.10 in W. Szymański [5], namely, $\pi$ is a quasinormal homomorphism if and only if each operator $\pi(s), s \in S$, is quasinormal and $\pi$ is a subnormal homomorphism.

(i) To prove that $\pi$ is subnormal we use the following criterion to characterize subnormal homomorphisms (W. Szymański [5]): $\pi$ is subnormal if and only if

$\Sigma(\pi(s+t) f(t), \pi(s+t) f(s)) \geq 0$ for all functions $f: S \rightarrow H$ with finite support.

Let $f$ be such a function. Then

$$
\begin{aligned}
\Sigma(\pi( & +t) f(t), \pi(s+t) f(s)) \\
& =\Sigma(\theta(s+t) \mu(s+t) f(t), \theta(s+t) \mu(s+t) f(s)) \\
& =\Sigma\left(\theta(s+t)^{*} \theta(s+t) \mu(s+t) f(t), \mu(s+t) f(s)\right) \\
& =\Sigma(\mu(s+t) f(t), \mu(s+t) f(s))=\Sigma(\mu(s+t) \mu(s+t) f(t), f(s)) \\
& =\Sigma(\mu(s+s+t+t) f(t), f(s))=\Sigma(\mu(s+s) \mu(t+t) f(t), f(s)) \\
& =\Sigma\left(\mu(s)^{2} \mu(t)^{2} f(t), f(s)\right)=\Sigma\left(\mu(t)^{2} f(t), \mu(s)^{2} f(s)\right)=\left\|\Sigma \mu(s)^{2} f(s)\right\|^{2} \geq 0 .
\end{aligned}
$$

Thus $\pi$ is subnormal.

(ii) Now we only need to prove that each $\pi(s), s \in S$, is quasinormal. Each $\pi(s), s \in S$, is subnormal, because $\pi$ is a subnormal homomorphism, as shown in (i) above. Hence each $\pi(s)$ is hyponormal, $s \in S$. A simple observation made by M. Embry in [1], page 63, that $T \in B(H)$ is quasinormal if and only if $T$ is hyponormal and $\left|T^{2}\right|=|T|^{2}$, completes the proof. Indeed, let $T=\pi(s), s \in S$. Since $\mu$ is a semigroup homomorphism, it follows that $\left|T^{2}\right|=|\pi(2 s)|=\mu(2 s)=$ $\mu(s)^{2}=|T|^{2}$.

\section{REFERENCES}

[1] M. Embry, A generalization of the Halmos-Bram criterion for subnormality, Acta. Math. (Szeged) 35 (1973), 61-64. MR 48:6994

[2] - The partially isometric factor of a semigroup, Indiana Univ. Math. Journal 32 (1983), 893-901. MR 86h:47059 
[3] A. Lubin, Weighted shifts and commuting normal extensions, J. Austral. Math. Soc. Ser. A 27 (1979), 17-26. MR 80j:47030

[4] B. Morrel and P. Muhly, Centered operators, Studia Mathematica 51 (1974), 251-263. MR 50:8132

[5] W. Szymański, Dilations and subnormality, Proc. Amer. Math. Soc. 101 (1987), 251-259. MR 88k:47062

Department of Mathematics, Millersville University, Millersville, Pennsylvania 17551

E-mail address: xcatepil@marauder.millersv.edu

Department of Mathematics, West Chester University, West Chester, Pennsylvania 19383

E-mail address: wszymans@wcupa.edu 